Compared to the control group, the CDK6 protein level was decreased in OCI-LY1/miR-320d group $(p<0.05)$. OCI-LY1/ miR-320d group and OCI-LY1/CDK6 shRNA group cells' proliferation ability were markedly decreased compared to the control group $(p<0.05)$. Relative luciferase activity value of experimental group is lower than that of the control group $(p<0.05)$.

Conclusions: Up-regulation of miR-320d expression can inhibit the ability of malignant proliferation of DLBCL cell. The mechanism is concerned with that miR-320d could inhibit the expression of target gene CDK6 at the post-transcriptional level.

\section{IMPROVED IMMUNOHISTOCHEMICAL METHOD FOR DETECTING ANTI-BCL-2 AND C-MYC ANTIBODIES IN MOUSE XENOGRAFT MODELS}

Xiaoling Xia, Jean Boyer, Liping Zhang, Burton Holmes, Rajalakshmy Ramalingam, Penny Towne Ventana Medical Systems, Roche, USA

Background: Antigen detection is known to be affected by preanalytical conditions such as fixative types, fixation time and delay to fixation. We used human breast carcinoma cell lines (BT-474 and ZR-75-1) generated xenograft tumors as model systems to analyze the impact of different pre-analytical conditions on BCL-2 and c-MYC staining. Antigen detection on mouse tissue is complicated by high level background staining due to the binding of secondary anti-mouse antibody to endogenous mouse tissue Igs and other components. We developed methods to use modified OptiView DAB detection system and unique linker methodology to almost completely eliminate non-specific staining and achieve satisfactory staining results.

Methods: Human breast carcinoma cell lines (BT-474 and ZR75-1) xenograft tumors were fixed across a range of times in 6 different commonly used fixatives as well as delay to fixation with a range from immediate fixation to a 24 hour delay to fixation in $10 \% \mathrm{NBF}$. Two new IHC staining methods were developed to stain either anti-BCL-2 (124), a mouse monoclonal antibody or anti-cMYC (Y69), a rabbit monoclonal antibody, to compare with standard OptiView DAB detection system on a Ventana automatic staining instrument. An Abcam monoclonal rabbit anti-mouse IgG was employed as linker followed by a modified OptiView DAB system to detect BCl-2 expression. We also used a modified OptiView DAB detection system to measure c-MYC expression.

Results: Antigen detection with indirect immunohistochemical methods is hampered by high background staining if the primary antibody is from the same species as the examined tissue. This high background was eliminated by using an Abcam monoclonal rabbit anti-mouse IgG as a linker followed by a modified OptiView DAB detection system. New methods ensured us to achieve a more precise understanding of protein expression level. The xenografts tissues fixed with zinc formalin and Z-5 showed equivalent staining to $10 \% \mathrm{NBF}$ after fixation for at least 6 hours. However, AFA, 95\% EtOH, Prefer fixative did not perform equivalently over the range of fixation times when compared to $10 \% \mathrm{NBF}$, regardless of fixation time.
This study also demonstrated degradation of BCL-2 and c-MYC antigenicity in response to delay in fixation if tissue was left unfixed at room temperature for more than two hours.

Conclusion: Our study recommended that the best fixatives were $10 \%$ NBF, Zinc fixative and Z-5 between 6 hours and 72 hours for both anti-BCL-2 and anti-c-MYC antibodies. In addition, the tissues should be fixed within 2 hours after tissue collection. New IHC methods yielded minimum reduction of BCL-2 and c-MYC specific signaling. Using this method, the tissue sections were remarkably free of the background staining that is typically seen in mouse tissues with mouse antibodies. Hence, this procedure provides an improved, high sensitive method for detecting protein expression level loss due to inappropriate pre-analytical sample treatment.

\section{RARE HISTOLOGICAL PATTERNS OF ANGIOFIBROMA OF SOFT TISSUE}

Yuichi Yamada, Hidetaka Yamamoto, Kenichi Kohashi, Yoshinao Oda

Department of Anatomic Pathology, Pathological Sciences, Graduate School of Medical Sciences, Kyushu University, Maidashi 3-1-1, Higashi-ku, Fukuoka, Japan

Background: Angiofibroma of soft tissue (AFST) is a rare benign soft tissue neoplasm, which is characterized by fibroblastic cytomorphology, prominent vascular pattern and novel fusion genes NCOA2-AHRR/AHRR-NCOA2 or GTF2I-NCOA2. AFSTs present a wide spectrum of morphology, making a challenge to diagnosis, thus we came to the cogitation to report the special histological features confirmed by genetic analysis.

Aims: The study aims to reveal the histological variety of AFST confirmed by genetic data.

Methods: We reviewed all the 274 cases diagnosed as solitary fibrous tumor/hemangiopericytoma (232 cases), undeterminated tumors of fibroblastic differentiation (36 cases) and recently diagnosed as AFST ( 6 cases), and picked up the 12 cases histologically compatible with AFST. All the 12 cases were genetically analyzed by RT-PCR method, and immunohistochemical stains were performed for available cases.

Results: Highlighted histological findings were as follows; amianthoid fibers, ossification, lymphoid follicles, lymphoid cuff, hemosiderin deposition, aggregate of foamy histiocytes, cystic change, necrosis, and hemorrhage. Immunohistochemically, the tumor cells were positive for EMA (4/10 cases), desmin (4/10 cases), CD163 (6/6 cases), CD68 (3/6 cases), estrogen receptor (7/7 cases), progesterone receptor (1/6 case), D2-40 (4/7 cases) and STAT6 (1/6 case, weak nuclear stain), but negative for CD34, alpha-smooth muscle actin, musclespecific actin, S-100 protein, pan-cytokeratin, beta-catenin, MDM2 and CDK4. AHRR-NCOA2 fusion gene was detected in 8 cases.

Conclusions: We revealed the unreported histological variation and immunohistochemical findings of AFST and confirmed them by genetic methods. It was suggested that AFST should be considered in the diagnosis of fibrous or fibrohistiocytic tumors with unspecified features. 